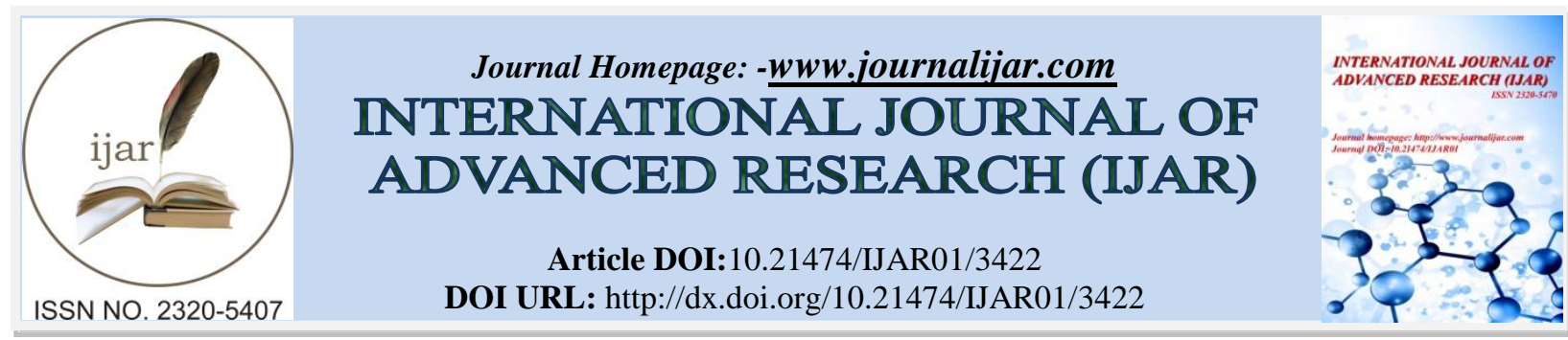

RESEARCH ARTICLE

\title{
OPTIMIZATION OF FORMULATION PARAMETERS AND CHARACTERIZATION OF SIMVASTATIN LOADED CHITOSAN NANOPARTICLES.
}

\author{
"N. Selvasudha M. Pharm¹, Dr. Kailasam Koumaravelou Ph.D. ${ }^{2}$, and N. Sri Durgadevi M.Pharm³ \\ 1. Research scholar, PRIST University, Puducherry campus,24/4, Uruvaiyar road, Abishegapakkam, Puducherry. \\ 2. Director, PRIST University, Puducherry campus,24/4, Uruvaiyar road,Abishegapakkam, Puducherry. \\ 3. Teaching fellow, Department of biotechnology, Anna University, Chennai.
}

\section{Manuscript Info}

Manuscript History

Received: 18 December 2016

Final Accepted: 15 January 2017

Published: February 2017

Key words:-

Nanoparticle; surfactant; stabilizer; poly vinyl alcohol; solvent evaporation simvastatin.

\begin{abstract}
This study aimed to optimize and characterize formulation factors in the preparation of drug-loaded polymeric nanoparticulate drug delivery system by employing response surface methodology (design of expertDOE). Nanoparticles were prepared by three different methods namely solvent evaporation, solvent diffusion, and cross-linking method. Effect of important factors like method of preparation, surfactant type, stabilizer type on particle size, and percentage entrapment efficiency were studied using DOE. The prepared formulations show particle size and $\%$ entrapment efficiency in the range of $602.11 \pm 12.5$ to 1005.55 $\pm 98.6 \mathrm{~nm}$ and $77.01 \pm 0.003$ to $97.01 \pm 0.21 \%$ respectively. Based on the result formulation prepared with nonionic surfactant (Tween 80), synthetic polymeric stabilizer (poly vinyl alcohol- PVA) and solvent evaporation method were found to be effective. After selection of optimized formulation, twelve formulations were prepared by altering key processing parameters like stabilizer and surfactant concentration and effective formulation was selected on the basis of in vitro release. Among various concentrations, formulation with combination of $0.50 \%$ w/v of PVA and $0.02 \%$ w/v of Tween 80 had sustain release property, stability and biocompatibility.
\end{abstract}

Copy Right, IJAR, 2017,. All rights reserved.

\section{Introduction:-}

Over the past decade, there has been a rise in particle manipulation and nanosizing of solute drug (simvastatin). The intrinsic shortcomings of conventional drug delivery and the potential of nanoparticles as drug delivery systems have offered incredible scope for researchers in the field of pharmaceuticals (Khan et al., 2013). Nanoparticles may be used for oral administration of gut-labile drugs or those with low aqueous solubility like simvastatin (Wong et al., 2010). These colloidal carriers have the ability to cross mucosal barrier as such. Besides, they have the potential for enhancing drug bioavailability via particle uptake mechanisms (Chen et al., 2011). It was therefore decided to prepare nanoparticles of simvastatin so as to optimize its delivery and overcome its inherent negative aspects like low solubility, low bioavailability, short half-life, dose-dependent adverse effect, etc. (PrakashKatakam et al., 2014 ). The success of formulation not only relies on the selection of technology but also based on the appropriate selection of polymer, surfactant, stabilizer, formulation parameters like stirring speed, temperature, etc.Nanoparticles are considerably easy to prepare but the stability and the selection of stabilizer(s) are the most challenging and critical step as they can affect drug bioavailability (Peltonen et al., 2010). Both polymers and 
surface active agents have been utilized as stabilizer for pharmaceutical nanoformulation. Polymers like PVP, PVA, HPMC , PEG, CMC (Mahesh et al., 2014, Danhier et al., 2014) and surface active agents like pluronic F68,F127, Tween 80, Tween 20, SLS, lecithin were used often in preparation. Stabilizer can be non-ionic or ionic in nature and the overall stability is based on electrostatic force. Single stabilizer may be sufficient but combinations of stabilizers are utilized for narrow distribution in particle size (Valo et al., 2013, George et al., 2013). In general, stabilizers are thought to be pharmaceutically inactive excipients and the selection of the stabilizers is performed keeping in mind only the physical stability (prevents particle aggregation). However, these stabilizers are not inactive. Many polymers and surfactants utilized as stabilizers for drug nanocrystals are affecting the cells and cell layers leading to modified bioavailability of active drug.

To optimize the formulation variables, RSM is used (collection of mathematical and analysis of problems) in which a response of interest is influenced by variables and the objective is to optimize this response (Sabir et al., 2000, Singh et al.,2005). Moreover RSM is helpful in rapid development of optimum formulation with minimum number of experiments for the investigation of influence of the independent variables on results (Roy et al., 2009, Jain et al., 2013). The objective of this research work was to formulate simvastatin nanoformulation using chitosan by three different methods (solvent evaporation, solvent diffusion, and cross-linking technique) and optimization of nanoformulation by response surface methodology (Mennini et al., 2008). Initially, preliminary trials were done with 1:1, 1:2, and 1:3 drug:polymer ratios for obtaining lag phase during drug release. With 1:2drug:polymer ratio, lag phase was obtained. Hence, this ratio was selected for the factorial design. During optimization, the effect of three independent variables, i.e., method of formulation (X1), stabilizer (X2), and surfactant(X3), on responses such as particle size (Y1) and \% entrapment efficiency (Y2) were studied. This study encompasses the development of further 12 formulations, which were analyzed by in vitro release characterization. Optimized formulation finally subjected to stability and biocompatibility studies.

\section{Experimental:-}

Materials:-

Simvastatin was obtained as gift sample from Biocon Pharmaceuticals. All chemicals and polymers used in this study were purchased from Sigma Aldrich and were of analytical grade.

\section{Preparation of nanoformulation:-}

Nanoparticles were prepared by solvent evaporation, solvent diffusion, and cross-linking technique (Krishna Sailaja et al., 2011).

\section{Solvent evaporation method:-}

The organic phase consisting of simvastatin (SS) solution (dissolved in methanol) was added drop wise to the aqueous solution containing polymer, surfactant and stabilizer under stirring condition at $400 \mathrm{rpm}$ for $3 \mathrm{hrs}$. Then it was homogenized at 10,000 rpm using Remi overhead stirrer. The formed nanoparticles were separated by centrifugation (REMI cooling centrifuge) at 20,000 rpm for $30 \mathrm{~min}$ followed by freeze-drying (Mao et al., 2008) .

\section{Solvent diffusion method:-}

The organic phase consisting of simvastatin (SS) solution (dispersed in dimethyl sulfoxide) was added dropwise to the aqueous solution containing polymer, surfactant, and stabilizer and kept in amagnetic stirrer at $400 \mathrm{rpm}$ for $3 \mathrm{hrs}$. Then it was homogenized at 10,000 rpm using Remi overhead stirrer. The formed nanoparticles were separated by centrifugation (REMI cooling centrifuge) at 20,000 rpm for $30 \mathrm{~min}$ followed by freeze-drying (Quintanar-Guerrero et al., 1996).

\section{Cross-linking method:-}

Methanol was added to simvastatin $(10 \mathrm{mg} / 5 \mathrm{ml})$, which was then incorporated to chitosan solution (20 mg dissolved in $5 \mathrm{ml}$ of $2 \%$ acetic acid). This organic phase was added to the aqueous solution containing polymer, surfactant and stabilizer under stirring at $400 \mathrm{rpm}$ for $3 \mathrm{~h}$ period. $150 \mu \mathrm{l}$ of $25 \%$ glutaraldehyde was added for cross-linking. Ethanolamine was finally added to block unreacted aldehyde group of cross linking agent (Murtaza et al., 2011). Then it was homogenized at 10,000 rpm using Remi overhead stirrer. The formed nanoparticles were separated by centrifugation (REMI cooling centrifuge) at 20,000 rpm for $30 \mathrm{~min}$ followed by freeze-drying. 
After optimization by factorial design,appropriate method of formulation, stabilizer,surfactant and concentration of additives were chosen by preparing various formulations from F25 to F36 as given in Table 2and characterized for its vitro release.

\section{Experimental design:-}

A central composite design having a unit value of $\alpha$ was applied according to reference protocols (Roy et al., 2009, Jain et al., 2013) to assess the influence of three independent variables (i.e., method of formulation, stabilizer and surfactant) on two dependent variables (i.e., particle size and encapsulation efficiency). All other formulations and process parameters were kept constant during the study. Stat-Ease design Expert®, version 7.0.3, was employed to generate and evaluate the statistical experimental design and construction of a design matrix with 24 experimental trials. This experimental trials of factorial design (table 1) consists of various combinations of three different methods, three types of polymeric stabilizers and surfactants coded as F1 to F24 as per Table 2. All the batches were prepared in triplicate $(n=3)$. The experiments were performed in random order. The response variables were evaluated by the following second-order polynomial model, as in Eq.1

$\mathrm{Y}=\beta \mathrm{o}+\beta 1 \mathrm{X} 1+\beta 2 \mathrm{X} 2+\beta 3 \mathrm{X} 1 \mathrm{X} 2+\beta 4 \mathrm{X} 12+\beta 5 \mathrm{X} 22 \ldots$. (1)

Where $\beta \mathrm{o}-\beta 5$ represents regression coefficients, $\mathrm{X} 1-\mathrm{X} 2$ the studied variables, and $\mathrm{Y}$ the measured response with each factor level combination. Different combinations were selected on the basis of the experimental design.

\section{Characterization:-}

Particle size analysis:-

Mean particle size and size distribution of simvastatin-loaded polymeric nanoparticles were determined by dynamic laser light scattering (SEM-633, SemaTech, France) at $25{ }^{\circ} \mathrm{C}$. The samples were diluted to an appropriate concentration using deionized water. Water was previously filtered through a $0.22-\mu \mathrm{m}$ membrane filter (Millipore, USA) to avoid the presence of any interfering particles. All measurements were carried out in triplicate; hence, each value is the mean of three independent readings within a batch and error represents the standard deviation of the mean particle size as an index of particle size and polydispersity.

\section{Transmission electron microscope:-}

About $5 \mu \mathrm{L}$ of nanoparticle suspension was deposited on a carbon film coated on a TEM copper grid and negatively stained with $2 \%$ uranyl acetate. The grid was tapped with a filter paper to remove extra water and then air-dried. The observation was done at an accelerating voltage of $100 \mathrm{kV}$ in a transmission electron microscope (Zeiss LEO906, Germany).

\section{Entrapment Efficiency of Nanoformulation:-}

Entrapment efficiency of nanoformulation was calculated by using the following equation

$\%$ Entrapment efficiency $=$ Total amount of drug added initially- free drug in supernatant/ Total amount of drug added initially $\times 100-------------------------E q .2$

A suspension of a representative nanoformulation was centrifuged at 12,000 rpm for 5 min until separation of all precipitate. The supernatant was collected for determination of drug content. It was then analyzed spectrophotometrically in triplicate at the wavelength of $238 \mathrm{~nm}$ (UV-visible spectrophotometer, Shimadzu UV1601, Japan). Calculation of simvastatin content was performed by using calibration curve method.

\section{In vitro drug release study:-}

In vitro release studies on formulation were carried out in PBS7.4 using dialysis bag technique. Then nanoformulation $(10 \mathrm{mg})$ was placed in $500 \mathrm{ml}$ dissolution medium and study was carried out for $24 \mathrm{~h}$. The temperature was maintained at $37.0 \pm 0.5{ }^{\circ} \mathrm{C}$ and the stirring speed at $100 \mathrm{rpm}$. Samples $(4 \mathrm{ml})$ were withdrawn and replaced with an equal volume of fresh dissolution medium at regular time interval and analyzed spectrophotometrically (model 1601, Shimadzu, Japan) at $238 \mathrm{~nm}$. The concentration of simvastatin in the samples was determined from a standard calibration curve. The release studies were carried out in triplicate.

\section{Forced degradation studies:-}

The ICH guideline entitled stability testing of new drug substances and products requires that stress testing be carried out to elucidate the inherent stability characteristics of the active substance. Stability studies of both simvastatin and formulation were carried out under extreme stress conditions like acidic, alkaline, hydrolytic, thermolytic, oxidation, photolytic (UV exposure) as per stability indicating assay methods (SIAM). 


\section{Acid degradation:-}

$0.1 \mathrm{~N} \mathrm{HCl}$ was taken in a $10 \mathrm{ml}$ volumetric flask and then accurately weighed $10 \mathrm{mg}$ sample was dissolved in it. To solubilize the drug, few drops of methanol was added and then the volume is made by $0.1 \mathrm{~N} \mathrm{HCl}$. Then, this solution was refluxed for 3days at room temperature in water bath. The specific amount of solution was withdrawn at end of each day. After this, the absorbance was measured by scanning the prepared solution of required concentration in a UV spectrophotometer (model 1601, Shimadzu, Japan).

\section{Alkali degradation:-}

$0.1 \mathrm{~N} \mathrm{NaOH}$ solution was prepared. Accurately weighed $10 \mathrm{mg}$ sample was taken in a $10 \mathrm{ml}$ volumetric flask. Then, the volume was made with $0.1 \mathrm{~N} \mathrm{NaOH}$. Then this solution was refluxed for 3 days at room temperature in a water bath. The absorbance was measured every day by withdrawing the required amount of the sample. Then, scanning was performed with a UV spectrophotometer.

\section{Degradation in pH solution:-}

Accurately weighed $10 \mathrm{mg}$ of drug was taken in a $10 \mathrm{ml}$ volumetric flask. Then, little amount of methanol was added to dissolve the drug. The volume was adjusted up to the mark with different $\mathrm{pH}$ solutions $(2,4,6,8,10)$. Then, that solution was refluxed for 3 days at room temperature in a water bath. The absorbance was measured at specific interval by withdrawing the required amount of sample solution. Then, scanning was performed with a UVspectrophotometer.

\section{Thermal degradation:-}

A specific amount of sample was taken in a clean petridish and dried, then the petridish along with drug was placed into the oven at $100{ }^{\circ} \mathrm{C}$ for 3days, $5 \mathrm{mg}$ of bulk drug was taken from the Petridish each day and $1000 \mathrm{ppm}$ solution with methanol was prepared. After this, the required concentration was made and the absorbance was measured in UV spectrophotometer.Percentage of degradation was calculated.

\section{Photolytic degradation:-}

Accurately weigh $10 \mathrm{mg}$ of sample in a $10 \mathrm{ml}$ volumetric flask and the volume was adjusted up to the mark with methanol. The prepared solution was placed in the photo stability chamber for 3 days. The absorbance was measured at end of each day by withdrawing the required amount of sample solution by using UVspectrophotometer.

\section{Oxidation with $\mathrm{H}_{2} \mathrm{O}_{2}$ :-}

$10 \mathrm{mg}$ of sample was weighed accurately, 2-3 drops of methanol was added to solubilise the drug. Then the volume was made with $3 \% \mathrm{H}_{2} \mathrm{O}_{2}$ and placed it in a cupboard for 3days.Specified amount of sample was taken and the required concentration was prepared each day. It was scanned in a UV spectrophotometer

\section{Hemolysis assay for biocompatibility:-}

Blood was collected from healthy adult volunteers and loaded into test tubes containing EDTA (anti coagulant) and diluted with PBS buffer.0.2 mL of diluted blood was added into fresh test tubes containing test samples (drug, polymer, two concentrations of formulation, positive and negative control) and incubated for $60 \mathrm{~min}$ at room temperature. $0.2 \mathrm{~mL}$ of diluted blood in $10 \mathrm{~mL}$ SLS and PBS buffer served as positive and negative controls respectively (HA etal., 2014).

\section{Results:-}

Appearance of nanoformulation:-

The nanoparticles were in size range of $602.11 \pm 12.5$ to $1005.55 \pm 98.6 \mathrm{~nm}$ (table 2) was confirmed by fig 3 . The TEM images in fig. 4 have shown the morphological properties and surface appearance of nanoparticles. The nanoparticles have nearly spherical shape and smooth surface. It also shows the homogenous molecular distribution of the drug in the polymer-based nanoparticles and drug distribution in the particulate form. There was no diffraction of transmission of electrons through the particles and that is why uniform dark particles were seen without any spot. Spotted particles support the presence of drug in particulate form rather than its distribution in molecular form.

The resultant equation for response-particle size $\mathrm{Y} 1$ is shown in Eq 3 and Figure 1.

$\mathrm{Y} 1=75.7281+3.3580 \mathrm{X} 1-1.9108 \mathrm{X} 2-0.2312 \mathrm{X} 1 \mathrm{X} 2-0.0198 \mathrm{X} 12-0.2109 \mathrm{X} 22$. 


\section{Drug entrapment efficiency:-}

Entrapment efficiency was in the range $77.01 \pm 0.003$ to $97.01 \pm 0.21 \%$ (table 2)and was dependent on the encapsulating polymer, stabilizer, and surfactant. RSM results for responseY2 (entrapment efficiency) is given in Figure 2. The resultant equation for response $\mathrm{Y} 2$ is given in Eq 4.

$\mathrm{Y} 2=27.047+1.087 \mathrm{X} 1+10.2130 \mathrm{X} 2+0.0475 \mathrm{X} 1 \mathrm{X} 2-0.0930 \mathrm{X} 12-0.8078 \mathrm{X} 22 \ldots \ldots$

\section{In vitro drug release study:-}

Drug release from the nanoformulation depends mainly on the type and concentration of the polymers used in the formulation. All the formulations displayed fastest to sustained release pattern with 67-90\% (table 3) released in PBS at $24 \mathrm{~h}$. The highest drug release is obtained for F33, F34, F35, and F36 in which both Tween 80 and PVA concentrations were higher. For formulation F25, F26, F27, and F28 (all contain 0.01\% Tween 80), there is no such difference, which indicates concentration of Tween 80 play a role in drug release profile. Among F29, F30, F31, and F32, the F29 containing the $0.5 \%$ concentration of PVA/0.02\% of Tween 80 showed the sustained drug release which is preferable for further drug development.

\section{Forced degradation study:-}

In this research, the nanosized chitosan-drug conjugates offers more stability as shown in Fig.5. Formulation minimally decomposed at acidic $\mathrm{pH}$ 2: however, the stability of this complex compared to simvastatin alone, for the same time interval, is much higher. At buffer above $\mathrm{pH} 6$, simvastatin is totally degraded. But the dissociation of formulation complexes decrease in higher (basic) $\mathrm{pH} 10.0$ (less than 5\% of complex degrade) indicating the extensive stability of simvastatin at alkaline $\mathrm{pH}$. The complexation event also protects simvastatin from the exposure to $\mathrm{UV}$ radiation $(\mathrm{O}=240 \mathrm{~nm})$ besides the damage by alkali. After a long exposure at short wavelength $\sim 40 \%$ of simvastatin molecules remains undamaged. At this condition, the uncomplexed or rather free simvastatin deteriorates $\sim 100 \%$. So the chitosan/stabilizer can reduce the degradation of active moiety due to protection of drug by H-bonding only to certain extent (as long as it holds) when compared to raw drug, but extensive protection is not possible. No oxidative degradation was noticed throughout the experiment which might be due to anti oxidant properties of both drug and polymer present in nanoformulation. When formulation was exposed to thermal stress, the results indicated that the formulation is less degraded and more stable compared to raw simvastatin. The thermal protection given by chitosan is reason for this stability.

\section{Hemolysis assay for biocompatibility:-}

Our results disclosed that all the tested concentrations of formulation neither showed hemolytic activity nor thrombus formation making it biocompatible for circulation in the blood. SLS which was used as a positive control showed 100\% hemolysis marked by complete lysis of the Red Blood Cells (RBC's) as shown in Fig. 6. While PBS which was used as the negative control, drug and nanoformulation did not show any hemolysis or toxicity to the RBC's making it a clinically suitable formulation.

\section{Discussion:-}

The main objective in this optimization study was to determine the experimental conditions which yield the best response including mean particle size around $500 \mathrm{~nm}, \%$ entrapment efficiency of about $95 \%$, uniform morphology and shape of the particles. Simvastatin was encapsulated by chitosan using an o/w emulsion solvent evaporation method. We studied the effect of three stabilizers on the characteristics of prepared nanoparticles, i.e., natural (xanthan gum), semisynthetic (carboxy methyl ethyl cellulose) and synthetic (PVA) and three surfactants, i.e., anionic (sodium lauryl sulfate), cationic (cetrimonium chloride), and nonionic (Tween 80), while other parameters such as drug concentration, polymer concentrations, stirring rate, temperature and the volume ratio of o/w phases were kept constant.

From the result of DOE, it was concluded that the formulation prepared by solvent evaporation method with nonionic surfactant (Tween 80 ) used at a concentration of $0.02 \% \mathrm{w} / \mathrm{v}$ and synthetic polymer PVA $(0.5 \%)$ was sufficient to facilitate the production of satisfactory nanoformulation. Attempts made in preliminary studies to use other surfactant types (except nonionic surfactant) failed to yield nanoparticles, but rather an aggregated mass was formed. Except synthetic polymer, other types of stabilizer resulted in decrease in the entrapment efficiency. Drug release from the formulation depends mainly on the type and concentration of additive used in the formulation. Formulation batches F25 and F29 which had a concentration 0.5\% of PVA relative to that of chitosan showed sustained release of drug over the $24 \mathrm{~h}$ period of the release study. Being biocompatible, PVA is widely employed in the fabrication of drug delivery systems. Due to its polar groups, it is an efficient proton acceptor; therefore, it can 
easily undergo hydrogen bonding with suitable compounds, especially polymers (chitosan) that behave as proton donors. The application of PVA as polymer matrix has been confirmed to possess significant outcomes, including enhancement of drug stability (Murtaza et al., 2011). Thus, simvastatin release was sustained from formulation F29 which contained a relatively accepted level of PVA. The nanoformulation with the highest concentration PVA showed fastest drug release in $\mathrm{pH} 7.4$ buffer.

\section{Effect of constant formulation parameters on nanoparticles formation:-}

The drug:polymer ratio, stirring rate and temperature were kept constant which also influence nanoparticles formation. Chitosan (encapsulating polymer) is a natural polymer (Riva et al., 2011, Patil et al., 2014) does not adversely affect the mucosal lining instead adhere to membrane and helps to rid gastrointestinal problems, and is commonly used for the development of oral drug delivery systems (Agnihotri et al., 2004, Ludwig et al., 2005 ). Its degradation is $\mathrm{pH}$ dependent, being sparingly soluble in acidic medium due to the presence of acidic group (Huanbutta et al., 2013Dudhani et al.,2010), but soluble in solution medium of $\mathrm{pH}>6.0$. It exhibits excellent swelling as a result of which it can retard drug release and is therefore used to achieve prolonged drug release (Dodane et al., 1998). This probably accounts for the very slow release of simvastatin from formulation F29 which contained a relatively optimum concentration of PVA and Tween 80. At this optimum concentration, polyelectrolyte complex formed between chitosan and PVA makes drug delivery effective in sustained release and higher entrapment efficiency with nanosize. Stirring rate also influenced the particle size and entrapment efficiency. The stirring speed of $400 \mathrm{rpm}$ at $37^{\circ} \mathrm{C}$ produced the nanoformulation of optimum size. By increasing stirring speed above $400 \mathrm{rpm}$, there was no nanoparticle yield (data not shown). This low yield could be due to the formation of smaller nanoparticle which was lost during washing process. Decreasing the stirring speed promotes aggregation of the nanoparticles and causes materials to adhere to the walls of beaker, thus resulting in low yield.

\section{Effect of method of preparation on encapsulation efficiency and particle size of the nanoformulation:-}

Among the three methods of preparation, the solvent evaporation method used for the preparation of the nanoformulation successfully entrapped simvastatin. The encapsulation efficiency for all nanoformulations prepared by $\mathrm{o} / \mathrm{w}$ emulsion-solvent evaporation technique was found higher. Simvastatin is practically insoluble in water; therefore, this substance was preferred partitioning into the dispersed organic phase of the emulsion. The amount of the drug passing into the aqueous phase was very low; thus, high encapsulation efficiencies were obtained (Reis CP et al., 2006).

Effect of PVA on encapsulation efficiency and particle size of the nanoformulation:-

During the solvent evaporation process, there is a gradual reduction in volume, resulting high viscous dispersed droplets. These affect the droplet size equilibrium, causing the coalescence and the agglomeration of the droplets during the early stages of the solvent removal (Abdel-Mottaleb et al.,2009). This problem can be managed by adding a steric stabilizer,e.g., PVA, into the continuous phase, thereby providing a thin protective layer around the droplets and hence reducing their coalescence (Juntanon et al., 2008). Using an insufficient quantity of stabilizer would result in large particles, but too much quantity would cause aggregation of the particles.

Three different concentrations of PVA with Tween in the outer aqueous phase were used in this study to find the best grade for production of nanoparticles. Effect of PVA concentration on the mean particle size of the produced nanoparticles shows that the concentration of PVA used plays an important role in determining the particle size of the obtained nanoparticles.

PVA with lower (data not shown) and higher concentrations yielded larger particles (d=1005.55 nm) in comparison to medium concentration $(538 \mathrm{~nm})$. PVA concentration in the external water phase is well known to influence the particle size of nanoparticle. Since the presence of PVA in the external phase stabilizes emulsion droplets against coalescence, increasing the PVA concentration usually leads to a decrease in the size of nanoformulation. However, we did not find any linear relationship between the PVA concentration and particle size. This might be due to the fact that higher concentration of hydrophilic polymer (PVA) makes particle to bombard leading to aggregation and larger particle size.

Particle sizes and morphology of the nanoparticles are given in fig 3 and 4. The particle size of formulations depends on viscosity of the dispersed phase which in turn directly related to the molecular weight of the polymer. As the energy level required to disperse even medium viscous solutions is too high, larger droplets formed during the emulsification process; as a result, greater nanoparticles were obtained when PVA concentration was increased. In 
spite of the iterative workup procedure applied to the nanoparticles including several washings with water, PVA of $0.5 \% \mathrm{w} / \mathrm{v}$ concentration covered the nanoparticle surfaces relatively more than the other three concentrations. The binding of PVA on the particle surface is likely to happen when the organic solvent is removed from the interface, in which interpenetration of PVA and chitosan molecules takes place. The hydrophobic vinyl acetate part of a partially hydrolyzed PVA serves as an anchoring site at theoil interface for binding to the surface of encapsulating polymer during the particle formation (Pillay et al.,2005). According to the results, it could be concluded that the greater the PVA concentration, the higher amount of residual emulsifier despite the several washing cycles. In addition, it induces local gelatinization of PVA and subsequent agglomeration of the nanoparticles, which might be responsible for the polydispersity of the nanoparticles. Hence, this may be the reason for the larger particle size obtained for PVA with higher concentration. Use of PVA with a low concentration prevented the local gelatinization of PVA at the surfaces of emulsion droplets and restricted the aggregation of nanoparticles considerably. In light of these results, $0.5 \%$ PVA was good steric stabilizer agent in the outer aqueous phase.

\section{Effect of Tween 80 on encapsulation efficiency and particle size of the nanoformulation:-}

Some studies have indicated that particles with superior topographical characteristics were obtained when Tween 80 was used as an emulsifier, instead of Tween 20 or Tween 40, independent of their concentrations. This might be due to better emulsification capability of Tween 80 as compared with that of Tween 20 or Tween 40 (Hoeller et al.,2009). In addition, the Tween 80 molecules acting as amphiphilic molecules deposited at the particle surface resulting in increment of particle size; moreover, they could shield surface charge of the nanoformulation led to decrease in zeta potential. Three different percentages $(0.01 \%, 0.02 \%$, and $0.03 \% \mathrm{w} / \mathrm{v})$ of Tween 80 in the external aqueous phase were selected according to the literature studies(Mitra et al., 2003,Prieto et al., 2013). It is known that the concentration of Tween in the external aqueous phase is an important key factor for the nanoparticles size. The effect of Tween 80 concentration on particle size and zeta potential of nanoformulation was investigated. According to the results, the mean particle size of the prepared nanoparticles was increased by increasing the Tween 80 concentration. Since nanoparticles were formed from the emulsion droplets after solvent evaporation, their size is intensely dependent upon the size and the stability of the emulsion droplets. Although an increase in size was observed when Tween concentration increased from 0.1 to $0.3 \%(\mathrm{w} / \mathrm{v})$, it was not significant above $0.4 \%$ (data not shown). The increased particle size nanoparticles at higher Tween 80 concentration is due to the further head group hydration resulting in greater emulsion droplets. These emulsion droplets are gradually solidified to form nanoparticles during the solvent evaporation process. Another reason for increased nanoparticle size may be due to higher emulsifier concentration which frequently results in increased viscosity of the outer phase, leading to highly aggregated droplets. This high viscosity leads to resistance toward shear forces in emulsion and less efficient stirring with a negative impact on the size of nanoparticles. According to the results, $0.2 \%$ (w/v) of Tween 80 concentration is the optimal value to obtain nanoparticles with appropriate particle size around $500 \mathrm{~nm}$.

It was found that the nanoparticles obtained from formulations containing $0.02 \% \mathrm{w} / \mathrm{v}$ Tween 80 could be suspended in solvent for $48 \mathrm{~h}$ before settling at the bottom of the test tube. This is due to deposition of Tween 80 on nanoformulation surface providing steric repulsion effect preventing agglomeration of the nanoparticles. Formulation with lower surfactant possessed more zeta potential, due to insufficient electrostatic repulsion to stabilize nanosuspensions. However, formulation with $0.03 \%$ Tween 80 possessed larger particle size but less zeta potential. It showed agglomeration and sedimentation of nanoparticles within $24 \mathrm{~h}$ as well. This implied that steric repulsion effect from Tween 80 could stabilize this system. Therefore, Tween 80 at the concentration of $0.02 \%$ w/v could be considered as a suitable concentration for stabilization of nanoparticles.

\section{Effect of PVA and Tween 80 concentration on drug release:-}

Among F29, F30, F31, and F32, the F29 containing the $0.5 \%$ concentration of PVA/0.025\% of Tween 80 showed the sustained drug release, which is preferable for further drug development.

Two possible mechanisms could explain the drug release from F29 formulation. These are swelling/pore formation from the spherical matrices and the matrix erosion resulting from degradation of nanoformulation (Fu et al., 2010). In actual circumference, since both PVA and chitosan is hydrophilic, the drug release in $24 \mathrm{~h}$ should be faster. In contrary to this, at particular combination, formulation shows sustained release. At this concentration of stabilizer and surfactant polyelectrolyte complex which offer intra hydrogelation properties, formed making slower drug release (Ravi Sankar et al., 2013, Garud et al., 2012). 


\section{Mechanism of stability produced by nanoformulation:-}

The oxidative degradation of drug substance involves an electron transfer mechanism to form reactive anions and cations. Amines, sulfides and phenols are susceptible to electron transfer oxidation to give N-oxides, hydroxylamine, sulfones and sulfoxide The functional group with labile hydrogen like benzylic carbon, allylic carbon, and tertiary carbon or $\alpha$-positions with respect to hetro atom is susceptible to oxidation to form hydro peroxides, hydroxide or ketone(Ngwa et al.,2010, Boccardi et al., 2005). In this study no degradation above the limit was reported. The reason attributed is the anti oxidant properties of simvastatin and chitosan evidenced by many literature. In addition the electrostatic bond formed between amino group of chitosan and hydroxyl group of simvastatin resist the oxidative stress producing more stable compound.

In general hydrolysis is a chemical process that includes decomposition of a chemical compound by reaction with water. Hydrolytic study under acidic, basic and different $\mathrm{pH}$ condition involves catalysis of ionizable functional groups present in the molecule which is in bonded state in the formulation resisting decomposition. Less degradation compared to pure drug might be due to breakage of bond at particular $\mathrm{pH}$ which also essential for sustain release of drug to produce its pharmacological actions.

Light stress conditions can induce photo oxidation by free radical mechanism. Functional groups like carbonyls, nitro aromatic, N-oxide, alkenes, aryl chlorides, weak $-\mathrm{C}-\mathrm{H}$ and $-\mathrm{O}-\mathrm{H}$ bonds, are likely to introduce drug photosensitivity (Alsante et al.,2003). Limited protection from chitosan is offered to formulation due to strong $-\mathrm{N}-\mathrm{H}$ bonds. But the polymer couldn't withstand more light stress condition, so protection from light must be ensured.

\section{Effect of PVA and tween 80 in stability of nanoformulation:-}

Development of nanosized particles builds high energy surfaces, which can turn to aggregation and Ostwald ripening, if stabilization is not at an efficient level.Smaller the particle size, the more efficient the stabilization. Stabilization is required for the formation of nanoparticles as well as for the long-term stability during storage. Nanoformulation are formed from a solidcore surrounded by a stabilizer layer and distinctive stabilizers are surfactants or polymers.

Since the drug candidate-simvastatin used here for nanoformulation are poorly soluble and hydrophobic, the stabilizers PVA and tween 80 tend to enhance the wetting and dissolution properties of the active drug. Stabilizers can either be non-ionic (tween 80) or polymeric (PVA) in nature, but the stability is based on the classical DLVOtheory reached either via steric hindrance or electrostatic forces.

Stabilization with Tween 80- non-ionic surfactants and PVA-polymer is based on the steric stabilization effect. Steric stabilization created in F29 formulation is based on the formation of a mechanical barrier, a steric layer, between the particles and it requires polymeric chains on the particle surfaces that are long enough which offered by PVA. Temperature and $\mathrm{pH}$ changes, during the drying, can affect molecular mobility and hence the efficiency of steric stabilization, which is maintained in our study (Kim et al., 2010). Electrostatic stabilization formed by combination of chitosan, PVA, drug and tween 80 is based on formation of repulsive Coulomb forces between the charged colloidal particles. The three fundamental requirements for an efficient stabilizer are firm attachment to the solid surface, high percentage of stabilizer coverage on the nanoparticle surfaces and hydrophilic/lipophilic balance of the stabilizer (here the balance between tween 80 and PVA chain lengths, is essential to anchor onto the nanoparticle surfaces) which are satisfactorily given by the appropriate concentration of stabilizers used in this formulation(Liu et al,, 2015).

\section{Conclusion:-}

This study using DOE showed the response of independent factors on dependent factors with the help of response surface plots and polynomial equation. Optimized formulation of simvastatin-loaded polymeric nanoparticles achieved higher encapsulation efficiency with smaller particle size which was prepared by solvent evaporation method, synthetic polymer and with nonionic surfactant. The investigation for effective concentration of stabilizer and surfactant showed that the F29 containing the 0.5\%concentration of PVA/0.02\% of Tween 80 showed the sustained drug release, higher stability and biocompatibility. 
Table 1:- Factorial design for optimization of simvastatin loaded polymeric nanoparticles.

\begin{tabular}{|l|l|l|l|}
\hline \multirow{2}{*}{ Factors (independent variables) } & \multicolumn{1}{|c|}{ Levels } & & \\
\cline { 2 - 4 } & -1 & 0 & 1 \\
\hline $\mathrm{X} 1 \quad$ Method of preparation & SE & SD & CL \\
\hline X2 Type of polymeric stabilizer & NL & SS & SY \\
\hline X3 Type of surfactant & AN & CA & NA \\
\hline Response (dependent variables) & Constraints & & \\
\hline Y1:PS (nm) & Minimize & & \\
\hline Y2:EE (\%) & Maximize & & \\
\hline
\end{tabular}

Abbreviations: SE-Solvent evaporation, SD-Solvent diffusion, CL-Cross linking, NL- Natural, SS-Semi synthetic, SY-Synthetic, AN-Anionic, CA-Cationic, NA-non ionic, PS-Particle size, nm- Nanometer, EE-encapsulation efficiency.

Table 2:- Particle size and \% entrapment efficiency of nano formulations.

\begin{tabular}{|l|l|l|}
\hline Formulation code & Particle size $(\mathrm{nm})^{*}$ & \multicolumn{1}{|c|}{ Encapsulation efficiency $(\%)^{*}$} \\
\hline F1 & $602.11 \pm 12.5$ & $94 . .2 \pm 0.03$ \\
\hline F2 & $800 \pm 19.5$ & $85.4 \pm 0.01$ \\
\hline F3 & $749.7 \pm 41.7$ & $91.70 \pm 0.34$ \\
\hline F4 & $823.34 \pm 23.89$ & $84.20 \pm 0.09$ \\
\hline F5 & $950 \pm 15.8$ & $77.0 \pm 0.01$ \\
\hline F6 & $1005.55 \pm 98.6$ & $73.10 \pm 0.001$ \\
\hline F7 & $595.89 \pm 34.8$ & $95.00 \pm 0.002$ \\
\hline F8 & $987.86 \pm 12.8$ & $79.23 \pm 0.03$ \\
\hline F9 & $826.78 \pm 56.9$ & $85.21 \pm 0.78$ \\
\hline F10 & $925 \pm 10.7$ & $80.00 \pm 0.07$ \\
\hline F11 & $976.9 \pm 50.0$ & $82.00 \pm 0.21$ \\
\hline F12 & $850.31 \pm 34.90$ & $81.00 \pm 0.008$ \\
\hline F13 & $756.33 \pm 21.0$ & $85.52 \pm 0.07$ \\
\hline F14 & $778.47 \pm 15.91$ & $84.11 \pm 0.11$ \\
\hline F15 & $1000.0 \pm 10.10$ & $79.00 \pm 0.09$ \\
\hline F16 & $524.32 \pm 34.67$ & $97.01 \pm 0.21$ \\
\hline F17 & $700.0 \pm 18.7$ & $90.07 \pm 0.32$ \\
\hline F18 & $751.45 \pm 10.0$ & $92.10 \pm 0.01$ \\
\hline F19 & $795.0 \pm 20.9$ & $90.08 \pm 0.021$ \\
\hline F20 & $695 \pm 3.4$ & $89.02 \pm 0.091$ \\
\hline F21 & $598 \pm 30.9$ & $87.34 \pm 0.11$ \\
\hline F22 & $700 \pm 54.7$ & $91.91 \pm 0.005$ \\
\hline F23 & $604 \pm 14.89$ & $77.01 \pm 0.003$ \\
\hline F24 & $645 \pm 10.1$ & $84.78 \pm 0.03$ \\
\hline
\end{tabular}

Notes:*All values are mean+ SD $(n=3)$. 
Table 3:- Cumulative percentage drug release of formulations with respect to surfactant and stabilizer concentrations at $24 \mathrm{~h}$.

\begin{tabular}{|l|l|l|l|}
\hline Formulation code & Stabilizer $(\% \mathrm{w} / \mathrm{v})$ & Surfactant $(\% \mathrm{w} / \mathrm{v})$ & \% \\
\hline F25 & 0.5 & 0.01 & $70.90 \pm 1.30$ \\
\hline F26 & 1.0 & 0.01 & $72.00 \pm 2.10$ \\
\hline F27 & 1.5 & 0.01 & $71.00 \pm 1.10$ \\
\hline F28 & 2.0 & 0.01 & $72.11 \pm 3.20$ \\
\hline F29 & 0.5 & 0.02 & $67.43 \pm 1.00$ \\
\hline F30 & 1.0 & 0.02 & $72.21 \pm 2.50$ \\
\hline F31 & 1.5 & 0.02 & $81.03 \pm 4.30$ \\
\hline F32 & 2.0 & 0.02 & $87.06 \pm 2.20$ \\
\hline F33 & 0.5 & 0.03 & $74.11 \pm 0.34$ \\
\hline F34 & 1.0 & 0.03 & $83.23 \pm 0.91$ \\
\hline F35 & 1.5 & 0.03 & $87.21 \pm 0.34$ \\
\hline F36 & 2.0 & 0.03 & $90.01 \pm 1.30$ \\
\hline
\end{tabular}

Notes:*All values are mean+ SD $(\mathbf{n}=3)$.

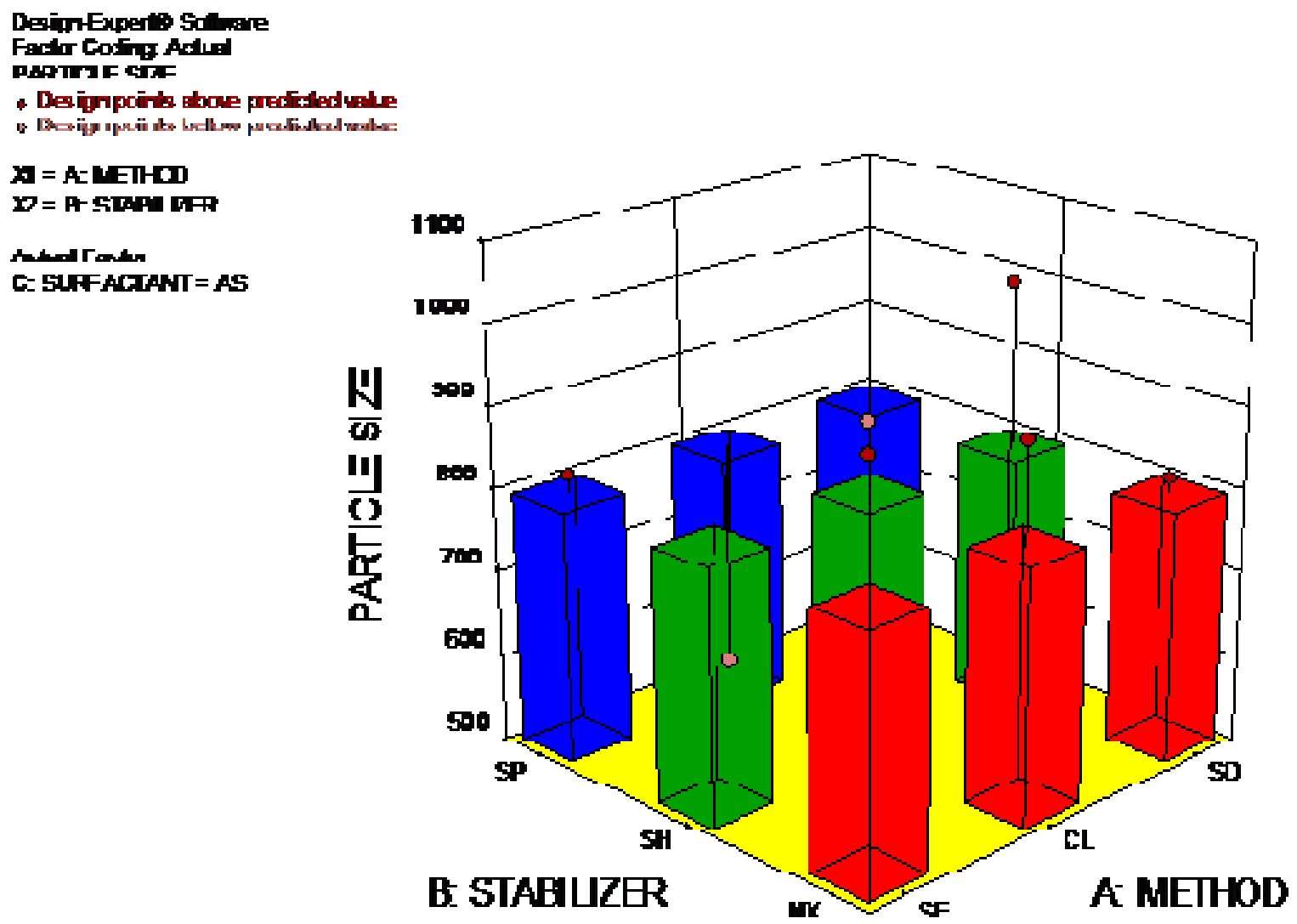

Fig.1 Response surface plot illustrating influence of method of preparations, polymeric stabilizers and surfactants on particle size 
D-ijExpiastal:

Turfir Cintenthe

EF (

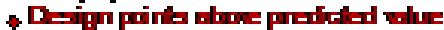

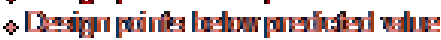

$\mathbf{X} 1=$ A $\mathbf{E T H O D}$

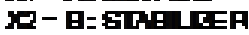

Andid Forter

C SARFATM = AS

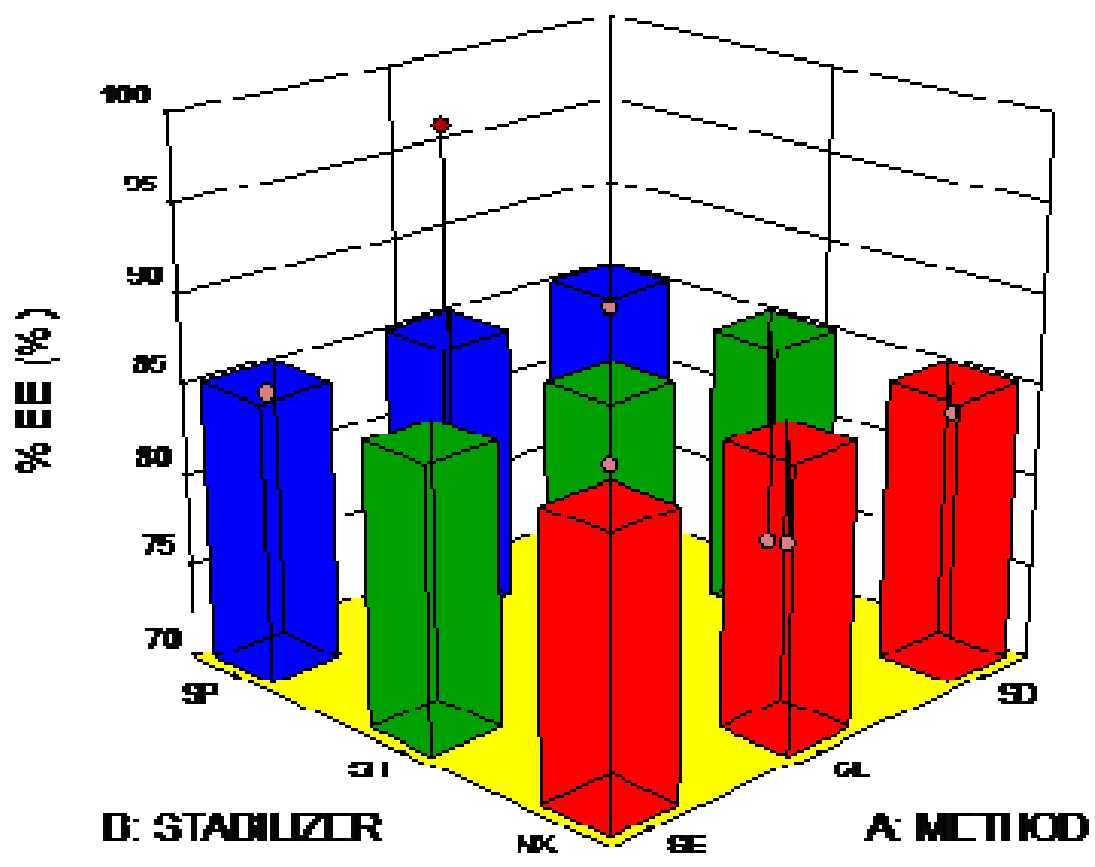

Fig.2 Response surface plot illustrating influence of method of preparations, polymeric stabilizers and surfactant on $\%$ encapsulation efficiency.

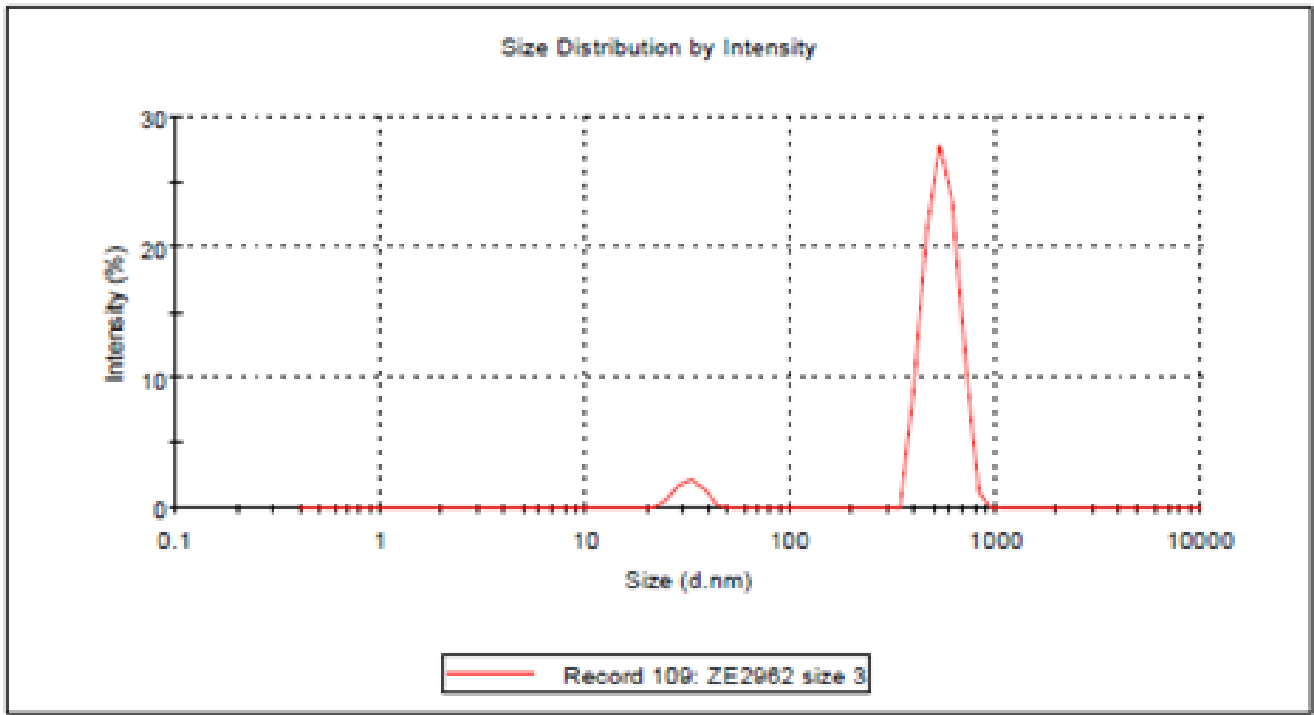

Fig.3 Particle size distribution curve of formulation showing nanosize range. 


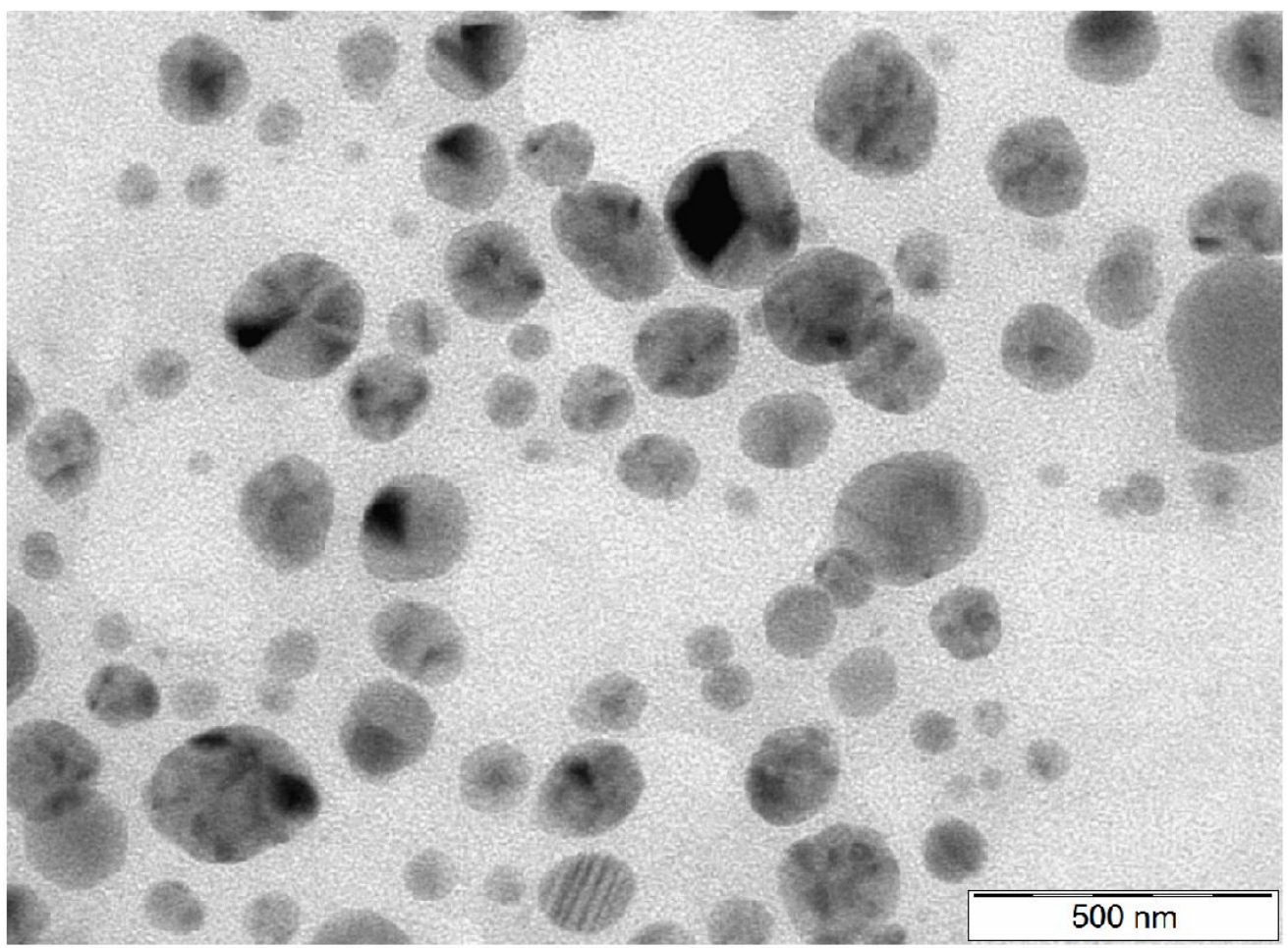

Fig.4 Transmission electron microscopy of formulation representing smooth, spherical, homogenious nanosized particles.

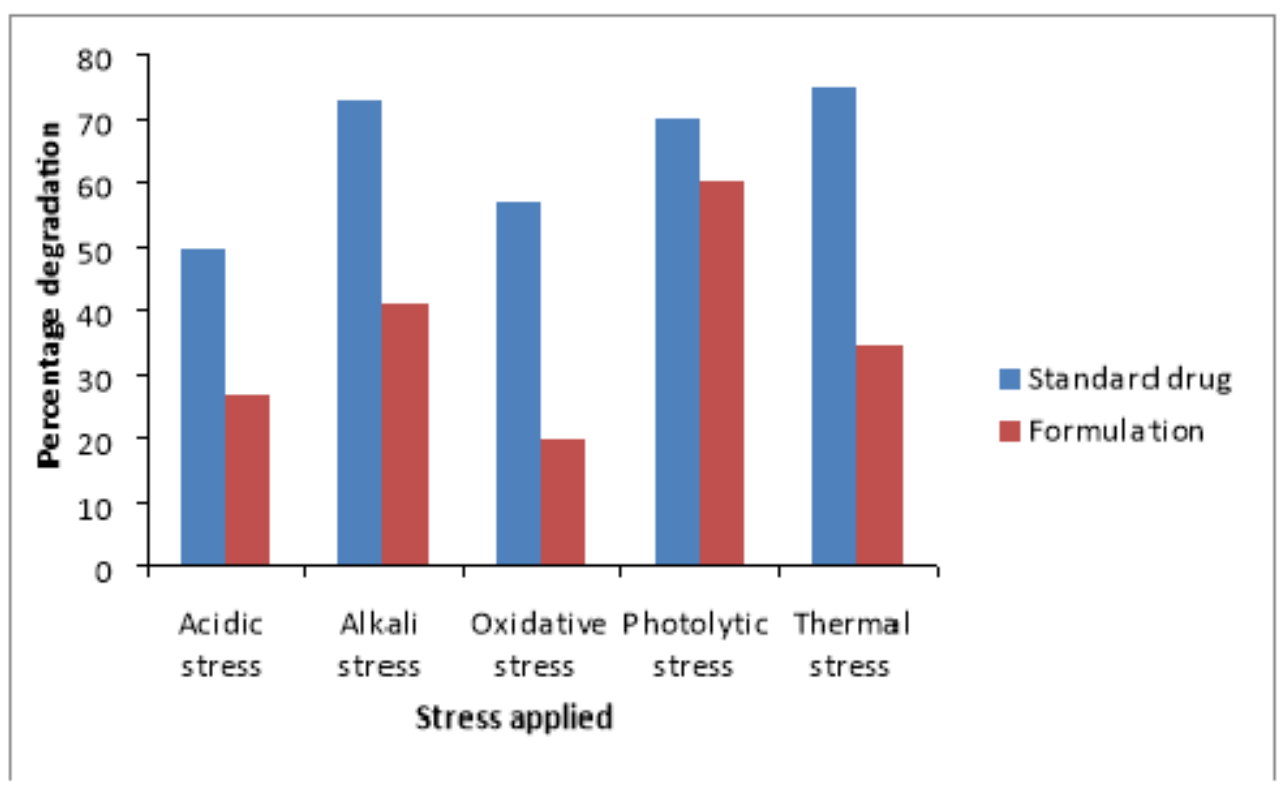

Fig. 5 Forced degradation study. Percentage degradation of drug and formulation when subjected to various stress conditions. 


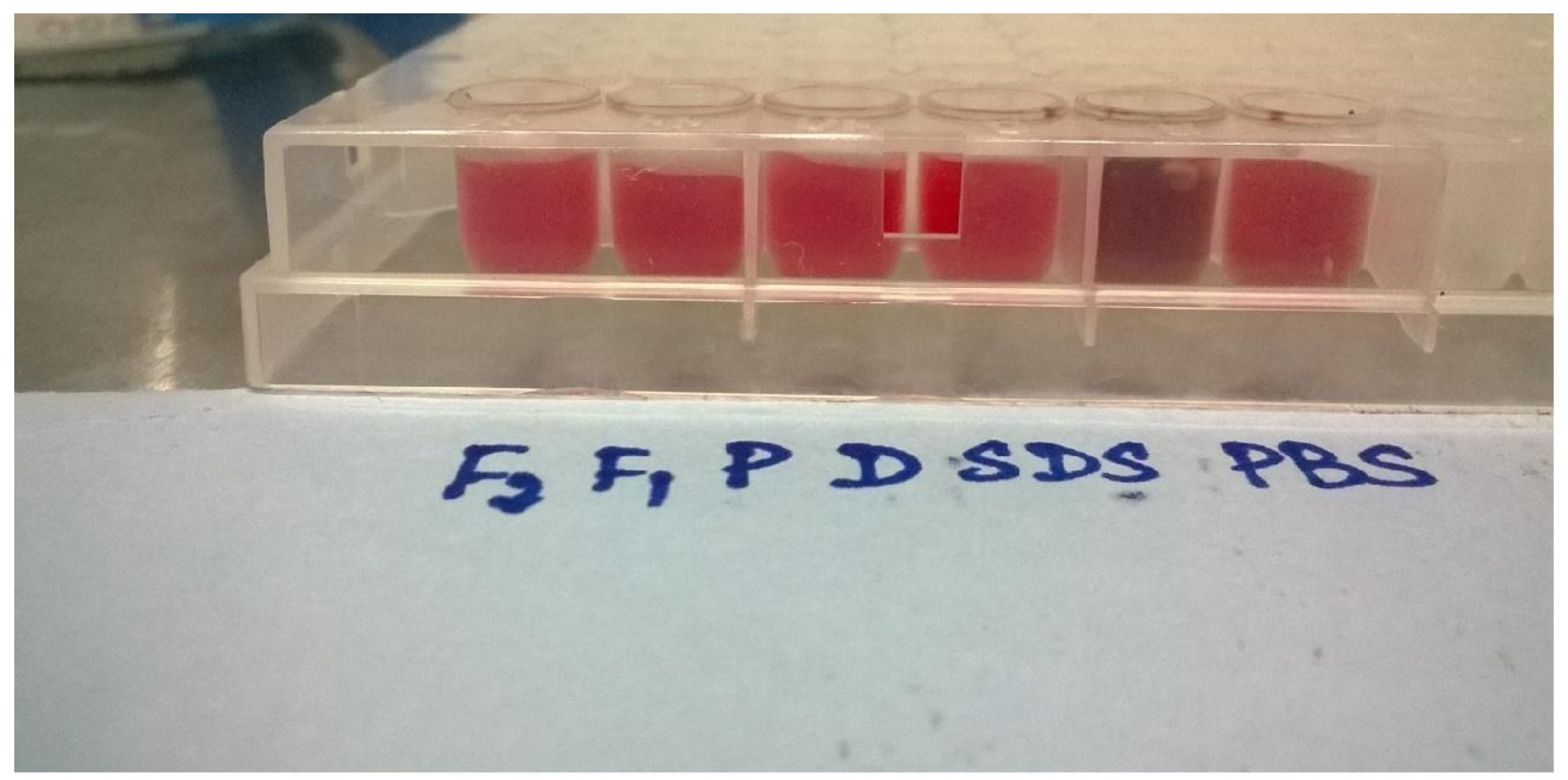

Fig. 6 Hemolysis assay

The biocompatibility of simvastatin loaded chitosan nanoparticles is shown with tubes from right to left as PBS-Phosphate saline buffer, SDS- sodium docecyl sulphate, D-Drug, P-Polymer, F1- Formulation with lowest concentration, F2-Formulation with highest concentration. All are showing biocompatibility when compared to positive control-SDS.

\section{Declaration of interest:-}

The authors state no conflict of interest and have received no payment in preparation of this manuscript.

\section{Acknowledgement:-}

Financial support from WOS-B 2011 scheme - Department of science and technology, Government of India (Ref.No. SSD/SS/O26/2011) is acknowledged

\section{References:-}

1. Abdel-MottalebMMA,Mortada ND, El-ShamyAA,Awad GAS.( 2009) Physically cross-linked polyvinyl alcohol for the topical delivery of fluconazole.DrugDevInd Pharm. 35(3), 311-320.

2. Agnihotri SA, MallikarjunaNN, Aminabhavi TM (2004 ) Recent advances on chitosan-based micro- and nanoparticles in drug delivery. J Controlled Release. 100, 5-28.

3. Alsante KM, Hatajik TD, Lohr LL, et al., (2003)Solving impurity/degradation problems: case studies S. Ahuja, K.M. Alsante (Eds.), Handbook of Isolation and Characterization of Impurities in Pharmaceutical, Academics Press, New York, p. 380

4. Boccardi G. (2005) Oxidative susceptibility testing S.W. Baertschi (Ed.), Pharmaceutical Stress TestingPredicting Drug Degradation, Taylor and Francis, New York p. 220.

5. Chawla V, Tiwary AK, Gupta S (2000) Characterization of polyvinylalcohol microspheres of diclofenac sodium: Application of statistical design. Drug DevInd Pharm. 26, 675-680.

6. Chen JP, Yang PC, Ma YH, Wu T (2011) Characterization of chitosan magnetic nanoparticles

7. for in situ delivery of tissue plasminogen activator. CarbohydrPolym. 84, 364-372.

8. Danhier, F.; Ucakar, B.; Vanderhaegen, M.-L.; Brewster, M.E.; Arien, T.; Préat, V (2014). Nanosuspension for the delivery of a poorly soluble anti-cancer kinase inhibitor. Eur. J. Pharm Biopharm. 88, 252-260.

9. Dodane V, Vilivalam VD (1998) Pharmaceutical applications of chitosan. PSTT.6, 246-253.

10. Dudhani AR, Kosaraju SL (2010) Bioadhesive chitosan nanoparticles: Preparation and characterization. Carbohydrate Polym. 81, 243-251.

11. Fu Y, Kao WJ (2010) Drug release kinetics and transport mechanisms of non-degradable and degradable polymeric delivery systems. Expert Opin Drug Deliv.7, 429-444.

12. Garud N, Garud A (2012) Preparation and in-vitro evaluation of metformin microspheres using non-aqueous solvent evaporation technique. Trop JPharmaceut Res. 11(4), 577-583. 
13. George, M.; Ghosh, I. (2013)Identifying the correlation between drug/stabilizer properties and critical quality attributes (CQAs) of nanosuspension formulation prepared by wet milling technology. Eur.J.Phar.Sci. 48(12):142-152.

14. H A SS, GPD C, Ravindran BR (2014) BSA Nanoparticle Loaded Atorvastatin Calcium - A New Facet for an Old Drug. PLoS ONE 9(2): e86317. doi:10.1371/journal.pone.0086317.

15. Hoeller S, Sperger A, Valenta C (2009) Lecithin based nanoemulsions: a comparative study of the influence of non-ionic surfactants and the cationic phytosphingosine on physicochemical behaviour and skin permeation.Int J Pharm. 370(1-2), 181-186.

16. Huanbutta K, Sriamornsak P, Luangtana-Anan M, Limmatvapirat S, Puttipipatkhachorn S, et al. (2013) Application of multiple stepwise spinning disk processing for the synthesis of poly(methyl acrylates) coated chitosan diclofenac sodium nanoparticles for colonic drug delivery. Eur J Pharm Sci. 50, 303-311.

17. Jain A, Jain SK (2013) Formulation and optimization of temozolomide nanoparticles by 3 factor 2 level factorial design. Biomatter. 3, 1-8.

18. JuntanonK,NiamlangS,RujiravanitR,Sirivat A (2008) Electrically controlled release of sulfosalicylic acid from crosslinked poly(vinyl alcohol) hydrogel. Int J Pharm. 356(1-2), 1-11.

19. KhanAA, MudassirJ,Mohtar N, Darwis Y (2013) Advanced drug delivery to the 680 lymphatic system: Lipidbased nanoformulations.Int J Nanomed. 681, 2733-2744.

20. Kim, S.; Lee, J (2010). Effective polymeric dispersants for vacuum, convection and freeze drying of drug nanosuspensions. Int. J. Pharm.397, 218-224.

21. Krishna Sailaja A, Amareshwar P, Chakravarty P (2011) Different techniques used for the preparation of nanoparticles using natural polymers and their application. Int J Pharm PharmSci. 3(2), 45-50.

22. Liu, P.; Viitala, T.; Kartal-Hodzic, A.; Liang, H.; Laaksonen, T.; Hirvonen, J.; Peltonen, L (2015) Interaction studies between indomethacin nanocrystals and PEO/PPO copolymer stabilizers. Pharm. Res.32, 628-639.

23. Ludwig A ( 2005) The use of mucoadhesive polymers in ocular drug delivery.AdvDrugDeliv Rev . 571, 595639.

24. Mahesh, K.V.; Singh, S.K.; Gulati, M (2014). A comparative study of top-down and bottom-up approaches for the preparation of nanosuspensions of glipizide. Powder Technol. 256, 436-449.

25. Mao S, Shi Y, Li L,et al (2008)Effects of process and formulation parameters on characteristics and internal morphology of poly(D,L-lactide-co-glycolide) microspheres formed by the solvent evaporation method.Eur $\mathbf{J}$ Pharm Biopharm. 68, 214-223.

26. Mennini N, Furlanetto S, Maestrelli F, Pinzauti S, Mura P (2008) Response surface methodology in the optimization of chitosan-Capectinate bead formulations. Eur J Pharm Sci. 35, 318-325.

27. Mitra A, Lin S (2003) Effect of surfactant on fabrication and characterization of paclitaxel-loaded polybutylcyanoacrylatenanoparticulate delivery systems J Pharm Pharmacol. 55, 895-902.

28. Murtaza G (2011) Development of glutaraldehydecrosslinked metronidazole loaded chitosan microcapsules: Analysis of dissolution data using DD solver. Latin Am J Pharm. 30, 1389-1395.

29. Ngwa G (2010) Forced degradation studies as an integral part of HPLC stability indicating method development Drug Deliv. Technol. 10 (5), pp. 56-59

30. Patil SS, Mohan Gupta VR, Gupta SK, Hiremath D (2014) Formulation and characterization of TPP crosslinked chitosan microspheres loaded with lornoxicam. J Biomed Pharm Res. 3,51-58.

31. Peltonen, L.; Hirvonen, J 2010 Pharmaceutical nanocrystals by nanomilling: Critical process parameters, particle fracturing and stabilization methods. J. Pharm. Pharmacol.62, 1569-1579.

32. PillayV,SibandaW,Danckwerts MP (2005) Sequential design of a novel PVA-based crosslinkedethylenichomopolymer for extended drug delivery. Int J Pharm. 301(1-2), 89-101.

33. PrakashKatakam, YadagiriPhalguna, DommatiHarinarayana (2014) Formulation, characterization and in vitro evaluation of capecitabine loaded polycaprolactone-chitosan nanospheres. Bangladesh PharmaceutJ.17(1), 1824.

34. Prieto C, Calvo L (2013) Performance of the biocompatible surfactant tween 80, for the formation of microemulsions suitable for new pharmaceutical processing. J ApplChem.2013, 1-10.

35. Quintanar-Guerrero D, Fessi H, Allémann E, et al (1996) Influence of stabilizing agents and preparative variables on the formation of poly(D,L-lactic acid) nanoparticles by an emulsification-diffusion technique. Int $\mathbf{J}$ Pharm. 143, 133-141.

36. Ravi Sankar V, Dhachinamoorthi D, Chandra Shekar KB( 2013) Formulation and evaluation of novel aspirin nanoparticles loaded suppositories. J PharmaceutSci. 13, 258-266.

37. Reis CP, Neufeld RJ, RibeiroAJ, Veiga F (2006) Nanoencapsulation I. Methods for preparation of drug-loaded polymeric nanoparticles.Nanomedicine. 2,8-21. 
38. Riva R, Ragelle H, Rieux A, Duhem N, Jerome C, Preat V (2011) Chitosan and chitosan derivatives in drug delivery and tissue engineering. AdvPolym Sci. 244, 19-44.

39. Roy P, Shahiwala A (2009) Statistical optimization of ranitidine $\mathrm{HCl}$ floating pulsatile delivery system for chronotherapy of nocturnal acid breakthrough. Eur J Pharm Sci. 37, 363-369.

40. Sabir A, Evans B, Jain S (2001) Formulation and process optimization to eliminate 735 picking from market image tablets.Int J Pharm. 215, 123-135.

41. Singh B, Kumar R, Ahuja N (2005) Optimizing drug delivery systems using 737 systematic "design of experiments". Part I: Fundamental aspects.CritRevTher Drug Carrier Syst. 22, 27-105.

42. Wong HL, Chattopadhyay N, Wu XY, Bendayan R (2010) Nanotechnology applications for improved delivery of antiretroviral drugs to the brain.Adv Drug Delivery Rev. 62, 503-517.

43. Valo, H.; Arola, S.; Laaksonen, P.; Torkkeli, M.; Peltonen, L.; Linder, M.B.; Serimaa, R.; Kuga, S.; Hirvonen, J.; Laaksonen, T (2013) Drug release from nanoparticles embedded in four different nanofibrillar cellulose aerogels. Eur. J. Pharm. Sci. 50, 69-77. 